\title{
ArcheoSciences
}

Revue d'archéométrie

$30 \mid 2006$

Varia

\section{Datation par thermoluminescence d'une structure de combustion granitique à Javols (Lozère) : quelques considérations sur la microdosimétrie des irradiations naturelles}

Thermoluminescence dating of a granitic fired structure at Javols (Lozère, France) : some inverstigations about natural radiation micro-dosimetry

Pierre Guibert, Saphir Grici, Françoise Bechtel, Floréal Daniel, Nicole Gourdon-Platel, Emmanuelle Vieillevigne et Alain Ferdière

\section{(2) OpenEdition Journals}

\section{Édition électronique}

URL : http://journals.openedition.org/archeosciences/263

DOI : $10.4000 /$ archeosciences. 263

ISBN : 978-2-7535-1595-6

ISSN : 2104-3728

Éditeur

Presses universitaires de Rennes

\section{Édition imprimée}

Date de publication : 31 décembre 2006

Pagination : 119-128

ISBN : 978-2-7535-0456-1

ISSN : 1960-1360

Référence électronique

Pierre Guibert, Saphir Grici, Françoise Bechtel, Floréal Daniel, Nicole Gourdon-Platel, Emmanuelle Vieillevigne et Alain Ferdière, «Datation par thermoluminescence d'une structure de combustion granitique à Javols (Lozère) : quelques considérations sur la microdosimétrie des irradiations naturelles », ArcheoSciences [En ligne], 30 | 2006, document 11, mis en ligne le 31 décembre 2008, consulté le 30 avril 2019. URL : http://journals.openedition.org/archeosciences/263 ; DOI : 10.4000/ archeosciences. 263 


\title{
Datation par thermoluminescence d'une structure de combustion granitique à Javols (Lozère) : quelques considérations sur la microdosimétrie des irradiations naturelles
}

\author{
Pierre Guibert*, Saphir Grici*, Françoise Bechtel*, Floréal Daniel*, \\ Nicole Gourdon-Platel*, Emmanuelle Vieillevigne* et Alain Ferdière**
}

\begin{abstract}
Résumé : Une structure de combustion creusée dans le granite sur les hauteurs dominant la ville romaine d'Anderitum, sur la commune de Javols (Lozère) a été datée par thermoluminescence. Pour cela, des grains de quartz de granulométrie comprise entre 80 et $315 \mu \mathrm{m}$ ont été extraits d'échantillons de la roche chauffée et analysés par thermoluminescence afin de déterminer la dose archéologique. Des mesures de spectrométrie gamma à bas bruit de fond au laboratoire et de spectrométrie gamma (NaI:Tl) sur site ont permis de déterminer la dose annuelle d'irradiation. En raison de l'hétérogénéité de la roche à l'échelle millimétrique, une étude spécifique de la répartition du potassium a été mise en œuvre, par spectrométrie X sous faisceau électronique d'un microscope électronique à balayage. Destinée à tester la représentativité de mesures globales de radioactivité par spectrométrie gamma vis-à-vis de l'irradiation bêta locale des grains de quartz retenus pour la TL, elle a montré que mesures globales et locales étaient en bon accord. L'âge moyen établi sur trois échantillons de roche est de $5825 \pm 280$ ans (1б) par rapport à 2004. La structure de combustion peut donc être rattachée à la période recouvrant le Néolithique final et le début du Chalcolithique.
\end{abstract}

\begin{abstract}
Thermoluminescence-dating has been applied to a fired pit dug into a granitic rock, on the hills at the vicinity of Anderitum, a gallo-roman city situated at Javols (Lozère, South of Massif Central, France). 80-315 $\mu \mathrm{m}$ size quartz grains were selected from samples of heated rock for equivalent dose determination. The annual dose was evaluated using low background gamma spectrometry (Ge detector) and on site NaI:Tl spectrometry measurements. At the millimetric size scale, Javols' granite is heterogeneous on the point of view of radioactive minerals and thus beta radiation field. It is the reason why a potassium distribution study (by Scanning electron microscopy-energy dispersive X ray spectrometry) was carried out in order to check for the appropriateness of global measures by gamma spectrometry compared to local beta irradiation given to quartz grains, the size of which is similar to that of the crystals studied by TL. We found that global and local measurements of potassium agree. The averaged age from three samples is $5825 \pm 280$ years (10) before 2004. Then, the fired structure can be chronologically linked to the late Neolithic and early Chalcolithic periods.
\end{abstract}

Mots clés : Datation, dosimétrie des irradiations naturelles, granite, quartz, thermoluminescence.

Key words: Dating, granite, natural radiation dosimetry, quartz, thermoluminescence.

Une structure de combustion, creusée dans un substrat granitique, a été mise au jour sur une colline surplombant le site gallo-romain de Javols en Lozère. Une attribution vraisemblable au Chalcolithique de cette structure a été proposée à partir de l'étude du seul tesson de céramique trouvé à l'intérieur de la fosse (Ferdière et al., 2006). Dans le but de

* IRAMAT-CRP2A UMR 5060 CNRS - Université de Bordeaux 3, Centre de Recherche en Physique Appliquée à l'Archéologie, Maison de l'Archéologie, Esplanade des Antilles, 33607 Pessac cedex. (guibert@u-bordeaux3.fr)

**UMR 6173 CITERES, Laboratoire Archéologie et Territoires, 35 allée Ferdinand-de-Lesseps, BP 60449, 37204 Tours cedex 03. 
tester cette hypothèse de rattachement chrono-culturel pour l'ensemble de la structure, nous avons appliqué la thermoluminescence à la datation du chauffage de la roche granitique. Du point de vue méthodologique, la nature même du matériau étudié, un granite, type de matériau peu utilisé dans notre communauté, offre l'opportunité d'une réflexion sur un problème actuel de la datation par luminescence, celui de l'hétérogénéité des irradiations bêta naturelles (à l'échelle millimétrique) auxquelles ont été soumis les minéraux sélectionnés pour la mesure de l'âge de chauffe.

\section{LE SITE ET LE MATÉRIEL ARCHÉOLOGIQUE}

Le site de Javols (Lozère) correspond à la ville romaine d'Anderitum, chef-lieu de la cité des Gabales (Gévaudan) à l'époque gallo-romaine. L'occupation la plus ancienne du site d'Anderitum ne paraît pas remonter au-delà du $\mathrm{II}^{\mathrm{e}}$ siècle avant J.-C. (Ferdière 2003), même si des traces antérieures y ont été décelées : silex taillés, apparemment néolithiques, dans diverses unités stratigraphiques des sondages, dans le vallon. Le paysage, granitique, est principalement constitué d'un vallon relativement large pour le secteur, occupé par la ville antique et surplombé, notamment à l'est et à l'ouest, de collines élevées (altitudes parfois supérieures à $1000 \mathrm{~m}$ ). Ces hauteurs sont, du côté occidental, formées d'un plateau assez étendu, aujourd'hui boisé (pins) et qui pourrait avoir constitué à la période pré-romaine un site d'oppidum, même si les sondages réalisés ici n’ont pas révélé d'indices déterminants dans ce sens : en effet, les colluvions en bas de pentes de ce côté comportent couramment du mobilier du $\mathrm{I}^{\mathrm{er}}$ siècle avant J.-C. et les quelques sondages réalisés ici en 2001 ont montré l'extrême érosion du plateau.

C'est à l'occasion d'une campagne de sondages systématiques sur ce plateau (Ferdière 2003; Ferdière et Ode 2001) qu'a été mise au jour la structure en question. Le sondage concerné a consisté au décapage de la surface du substrat granitique, ici peu profond (une dizaine de centimètres sous l'humus forestier), sur quelques mètres carrés. Notons dès à présent qu'aucune autre structure nette et/ou datable archéologiquement n'a été mise au jour dans ce secteur. Il s'agit d'une fosse allongée $(1,40 \times 0,45 \mathrm{~m})$ arrondie aux extrémités, d'axe approximativement est-ouest et creusée dans le substrat sur une profondeur d'environ $0,35 \mathrm{~m}$; le fond est plat, les parois des grands côtés quasi verticales et celles des petits côtés fortement inclinées.

Le matériel d'étude est constitué de prélèvements de granite, chauffé ou non, ainsi que du sédiment qui recouvrait la roche, que nous considèrerons semblable à celui qui comblait la fosse avant la fouille. Ces échantillons (Tableau 1) sont destinés à la datation ou à la caractérisation (nature, répartition, granulométrie, état d'altération des minéraux constitutifs et compositions chimique et radiochimique de la roche globale).

\section{Principe de la méthode de datation PAR THERMOLUMINESCENCE}

La plupart des solides isolants de nature cristalline, au cours d'un chauffage émettent de la lumière après avoir été

\begin{tabular}{|c|c|c|c|}
\hline Échantillons & Lieu de prélèvement & Caractéristiques & études effectuées \\
\hline BDX 8449 & Paroi nord de la fosse & Bloc rubéfié délité à $4 \mathrm{~cm}$ de la surface & Datation, caractérisation \\
\hline BDX 8451 & Paroi nord de la fosse & Un seul fragment rubéfié de $3 \mathrm{~cm}$ & Datation caractérisation \\
\hline BDX 8452 & Paroi nord de la fosse & Un bloc rubéfié de $5 \mathrm{~cm}$ & Datation caractérisation \\
\hline BDX 7970 & $\begin{array}{l}\text { À l'intérieur de la fosse (envoi d'A. Ferdière } \\
\text { pour tests préliminaires) }\end{array}$ & Retiré d'un bloc extrait de la fosse rubéfiée & $\begin{array}{l}\text { Caractérisation, } \\
\text { Test databilité }\end{array}$ \\
\hline BDX 8454 & $\begin{array}{l}\text { Terre de remplissage dans zone de surface } \\
\text { (sous végétation). }\end{array}$ & Non chauffé & Mesure radiochimie \\
\hline BDX 8455 & \multirow{2}{*}{$\begin{array}{l}\text { fragments de granite résultant du creusement } \\
\text { d'une galerie gamma-métrique à proximité de } \\
\text { la fosse rubéfiée }\end{array}$} & $\begin{array}{l}\text { Non chauffé, prélevé à } 10 \mathrm{~cm} \text { de profondeur } \\
\text { dans la galerie }\end{array}$ & Caractérisation \\
\hline BDX 8456 & & $\begin{array}{l}\text { Non chauffé, second prélèvement dans la } \\
\text { galerie }\end{array}$ & Mesure radiochimie \\
\hline
\end{tabular}

Tableau 1 : Site de Javols (Lozère), principales caractéristiques des échantillons et études entreprises. À l'exception de BDX 7970, tous les échantillons ont été collectés sur site par deux d'entre nous (FB et PG) en juillet 2003.

Table 1: Site of Javols (Lozère, France) samples and studies to be carried out. Except BDX 7970, all samples were collected by two of us (FB and $P G)$ in July 2003. 
au préalable irradiés. La méthode de datation par thermoluminescence exploite ces propriétés (Aitken, 1985).

Depuis sa formation, le matériau est bombardé par des particules $\alpha$ et $\beta$ ainsi que des rayonnements $\gamma$ et cosmiques. Sous l'effet de cette irradiation, le solide est ionisé et des électrons sont éjectés des atomes. La plupart retourne à l'état fondamental mais certains se retrouvent capturés durablement par des centres pièges, correspondant à des défauts à l'échelle atomique du matériau irradié. Pour extraire les électrons de ces centres pièges, il est nécessaire d'apporter de l'énergie au solide sous forme de chaleur, par exemple. Les électrons capturés par les pièges passent dans la bande de conduction et ils peuvent alors se recombiner avec des trous piégés au niveau de centres dits de recombinaison. Ces centres peuvent émettre des photons caractéristiques au cours de ce processus : c'est la thermoluminescence.

Au bilan, le chauffage ancien du matériau permet de remettre à zéro les centres pièges, qui tendent à se remplir progressivement sous l'action de la radioactivité naturelle, une fois le matériau refroidi. L'étude au laboratoire, quelques milliers d'années plus tard, reproduit le chauffage ancien, mais cette fois-ci la luminescence est mesurée. Nous pouvons donc déterminer la quantité d'énergie absorbée par les cristaux depuis le chauffage ancien, en d'autres termes nous pouvons déterminer la dose archéologique par comparaison avec la luminescence produite sur les mêmes cristaux par une irradiation calibrée artificielle au laboratoire.

En plus de la dose archéologique, il est nécessaire de déterminer la dose annuelle d'irradiation, énergie absorbée par les cristaux en une année, grâce à des mesures de radioactivité sur le terrain et au laboratoire. Nous ajouterons que l'on suppose que la dose annuelle a été constante depuis le moment du chauffage. Dans la mesure où une évolution de la compostion radiochimique de l'échantillon et du milieu d'enfouissement est un phénomène assez fréquent, le débit de dose annuel est susceptible d'évoluer également avec le temps et ce qui importe c'est d'en déterminer la valeur moyenne. Nous détaillerons cet aspect plus loin. Avec ces deux grandeurs expérimentales, dose archéologique et dose annuelle moyenne, il est finalement possible d'obtenir l'âge de l'échantillon à compter de sa dernière cuisson, par la relation suivante :

âge TL(a)= Dose archéologique (Gy)/ Dose annuelle (Gy/a) [Eq. 1]

où Gy est le symbole du Gray, unité de dose absorbée (1 Gy équivaut à une énergie absorbée de 1 Joule/kg).

La dose annuelle, notée I, est composée de contributions distinctes par la nature et la portée des particules (alpha et bêta) ou des rayonnements (gamma et cosmiques) ionisants. Elle s'exprime selon la relation :

$\mathrm{I}=\mathrm{kI}_{\alpha}+\mathrm{I}_{\beta}+\mathrm{I}_{\gamma}+\mathrm{I}_{\text {cosmique }}[$ Eq. 2]

Le facteur $k$, déterminé par des expériences de TL, pondère la contribution alpha (énergie déposée annuellement par les alpha) en raison du fait que ces particules ont une efficacité en thermoluminescence beaucoup plus faible que les particules $\beta$ ou les photons $\gamma$. D'autre part, il y a pour les contributions alpha et bêta une dépendance avec la dimension des grains sur laquelle nous reviendrons.

\section{3. ÉTUDE DE LA THERMOLUMINESCENCE}

La méthodologie suivie en TL dépendant étroitement de la nature du matériau étudié, nous avons jugé utile de présenter certains aspects expérimentaux conduisant à la mesure de dose, dans le cadre spécifique de ce travail.

\subsection{Préparation du matériau}

Pour la détermination de la dose archéologique nous avons choisi de travailler exclusivement sur les quartz en raison des risques de fading anomal (anomalous fading ou perte anormale de TL) des feldspaths pouvant conduire à une sous-estimation de l'âge (Wintle, 1973; Sanderson, 1988; Zink, 1996). La granulométrie choisie, entre 80 et $315 \mu \mathrm{m}$, et la friabilité du granite chauffé permettaient une extraction aisée des cristaux, bien que le rendement se soit avéré faible par la suite (moins de $1 \%$ en poids de l'échantillon était constitué de quartz de cette classe granulométrique). Néanmoins, les quantités extraites ont été suffisantes pour mener à bien les datations.

Le protocole de séparation des quartz a été adapté d'une technique utilisée dans la communauté de la datation (Fleming, 1970, 1978; Mejdahl, 1982; Mejdahl et WinterNielsen, 1982, Hutt et Smirnov, 1983), et à chaque étape nous en avons vérifié l'avancement par des enregistrements de diffraction de rayons X. Après attaque chlorhydrique $(\mathrm{HCl} 1 \mathrm{M})$ des grains, qui est pratiquée systématiquement pour éliminer les éventuels carbonates, la fraction de densité comprise entre 2,62 et 2,68 est sélectionnée par flottation dans des solutions denses de polytungstate de sodium. Cette fraction, d'après les diagrammes $X$ contenait essentiellement quartz (densité 2,65) et albite. L'élimination totale des feldspaths résiduels a été conduite par attaque à l'acide hexafluorosilicique $\left(\mathrm{H}_{2} \mathrm{SiF}_{6} 31 \%\right.$ durant 64 heures$)$. 


\subsection{Protocole de mesure de la dose archéologique}

Le protocole de mesure de la dose archéologique utilisé correspond à une méthodologie validée et fiable : méthode des « ajouts de doses et de régénération " dont les principes ont été exposés dès le début des années 1970 (Zimmermann, 1971) et la méthodologie affinée par la suite (Guibert et al., 1996; Sanzelle et al., 1996; Roque et al., 2004a, b). Elle consiste, sur une première série de prises d'essais identiques (de quartz) à enregistrer d'une part les courbes de TL naturelle (provoquée par l'irradiation naturelle depuis le dernier chauffage que l'on cherche à dater) et, d'autre part, à enregistrer le signal de TL obtenu après irradiations préalables par des doses connues (on ajoute donc une dose artificielle à la dose naturelle). Cet ensemble de courbes obtenues par ajout de dose (Fig. 1) ne suffit pas à la mesure de la dose archéologique. Pour mener à bien cette détermination, il est nécessaire de connaitre le comportement du matériau à

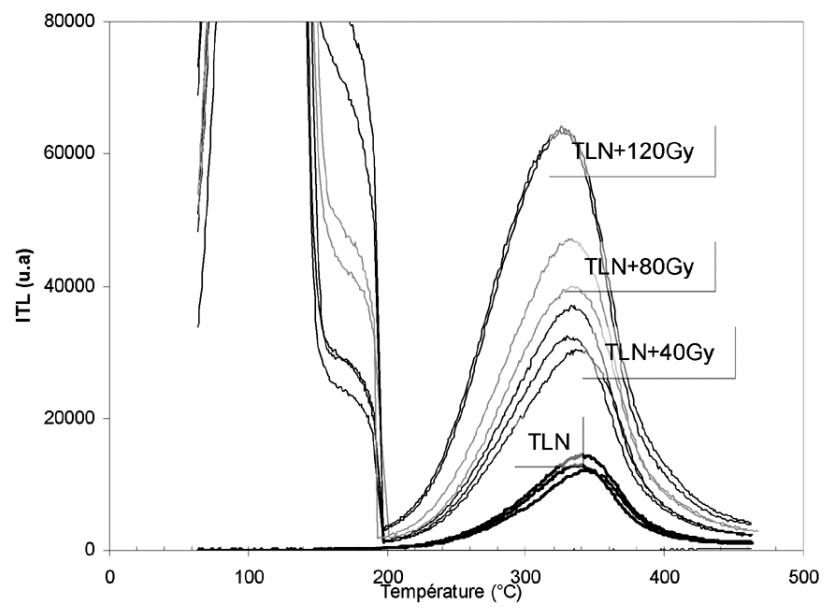

Figure 1 : Courbes de thermoluminescence de première lecture de l'échantillon de granite chauffé BDX 8449 (TLN : thermoluminescence naturelle). Le signal d'intérêt pour la datation se situe à des températures supérieures à $200^{\circ} \mathrm{C}$. Conditions expérimentales : vitesse de chauffe $4{ }^{\circ} \mathrm{C} / \mathrm{s}$ sous flux d'azote, préchauffage à $200{ }^{\circ} \mathrm{C}$ pendant 2 minutes, filtrage optique : 2 filtres Schott BG12 et 1 filtre réflecteur d'infrarouge MTO Ta3, tube PM : EMI 9813 QKA, bande passante : 350-450nm. Irradiation bêta par source céramique ${ }^{90} \mathrm{Sr} /{ }^{90} \mathrm{Y}$ AEA Technology type SIF 1176 délivrant $0,0737 \pm 0,0015 \mathrm{~Gy} / \mathrm{s}$ à la date des analyses.

Figure 1: First reading TL curves of quartz extracted from sample BDX 8449 (TLN: Natural TL). The region of interest for dating is situated above $200{ }^{\circ} \mathrm{C}$. Experimental details: Home made TL reader, heating rate $4^{\circ} \mathrm{C} / \mathrm{s}$, preheat at $200{ }^{\circ} \mathrm{C}$ for 2 minutes, 2 Schott $B G 12$ and 1 MTO Ta3 IR rejector optical filters, EMI 9813 QKA PMT, global spectral window from 350 to $450 \mathrm{~nm}$, beta radiation (dose rate: $0.0737 \pm 0.0015 \mathrm{~Gy} / \mathrm{s}$ in March 2004) delivered by a ceramic type beta source ${ }^{90}$ Sr ${ }^{90}$ Y AEA Technology SIF 1176. l'irradiation tel qu'il était à l'issue du chauffage archéologique. Ainsi, de nouvelles expériences de TL, dites de seconde lecture ou "régénérées ", sont entreprises sur une seconde série de prises d'essai. Les quartz utilisés pour cette étape sont issus de la même préparation que les précédents, la différence étant qu'ils ont été « vidés » de leur thermoluminescence naturelle par un traitement thermique préalable à leur irradiation au laboratoire et la lecture consécutive de leur TL. Cette étape est importante, car la qualité et la fiabilité $\mathrm{du}$ résultat de datation dépendent des conditions de recuit (Roque et al., 2004a,b). Les conditions de recuit dépendant de l'histoire thermique de chaque échantillon doivent être déterminées au cas par cas. Cela est réalisé à partir d'autres expériences de TL basées sur la similitude des courbes de TL obtenues après recuits à différentes températures suivis d'irradiation et des courbes de TL naturelle. Nous retenons les conditions de recuit qui permettent d'obtenir les courbes de TL les plus homothétiques (Vieillevigne et al., 2004).

\subsection{Détermination de la dose archéologique : les résultats}

Le traitement des données expérimentales est classique. Il comprend dans une première étape le test du plateau qui consiste à rechercher le domaine de stabilité thermique des signaux de TL : à Bordeaux, on utilise le plateau de dose (Roque et al., 2004b), pour lequel la dose archéologique est calculée point par point; on ne retient des courbes de TL que le domaine de température pour lequel la dose calculée atteint une valeur constante aux incertitudes de mesure près. Puis chaque courbe est intégére dans ce domaine. Le traitement de ces données permet de déterminer les lois de croissance de la TL avec la dose, d'où l'on déduit la dose archéologique finale comme illustré par la figure 2. Pour les quartz du granite de Javols, le domaine retenu s'étend de 340 à $420-450^{\circ} \mathrm{C}$ selon l'échantillon.

Les résultats des mesures de TL sont présentés au tableau 2. Les conditions de recuit équivalent ( 1 heure à la température indiquée) ont permis pour chaque échantillon de remettre à zéro leur thermoluminescence et de reproduire les propriétés de luminescence acquises à l'issue du chauffage archéologique. Nous noterons que cette température de recuit ne correspond pas exactement à la température, nécessairement supérieure, à laquelle furent portés les cristaux dans le passé. Elle correspond aux conditions thermiques qui permettent de retrouver ou de se rapprocher le plus de l'état électronique des centres-pièges dits "très profonds " des cristaux naturels à l'issue de leur chauffage ancien (Roque et al., 2004b; Duttine et al., 2005). 


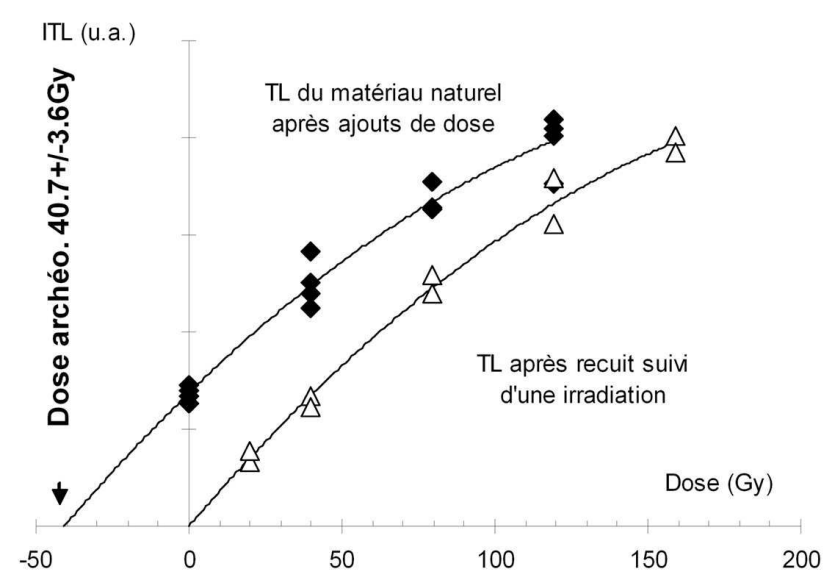

Figure 2 : Après intégration des signaux de TL entre 320 et $420^{\circ} \mathrm{C}$, la loi d'acquisition de la TL donne une dose archéologique avoisinant 40 Gy pour l'échantillon de granite BDX 8449.

Figure 2: Growth curves of TL signals versus laboratory dose after $T L$ has been integrated between 340 and $420^{\circ} \mathrm{C}$ (plateau region). The additive dosel annealing/ regeneration (ADAR) Technique was employed (Roque et al., 2004a) and data processed according to a slide method described by Guibert et al. (1996).

\begin{tabular}{|c|c|c|}
\hline Échantillon & $\begin{array}{c}\text { Température de } \\
\text { recuit }\end{array}$ & Dose archéologique \\
\hline BDX 8449 & $350^{\circ} \mathrm{C}$ & $40,7 \pm 3,6 \mathrm{~Gy}$ \\
\hline BDX 8451 & $325^{\circ} \mathrm{C}$ & $33,1 \pm 1,4 \mathrm{~Gy}$ \\
\hline BDX 8452 & $400^{\circ} \mathrm{C}$ & $34,4 \pm 2,9 \mathrm{~Gy}$ \\
\hline
\end{tabular}

Tableau 2 : Granite chauffé de Javols, résultats de l'étude de thermoluminescence : température de recuit et dose archéologique. Table 2: Heated granite samples from Javols, thermoluminescence results: annealing temperature (for $1 \mathrm{~h}$ ) and equivalent dose.

\subsection{Détermination du coefficient d'efficacité alpha}

Compte tenu de la dimension des grains $(80-315 \mu \mathrm{m})$, largement supérieure aux parcours des particules alpha, le signal TL obtenu ne contient qu'une contribution réduite, cependant non négligée, provenant de l'irradiation alpha par les minéraux présents au voisinage des grains de quartz. On a supposé comme il est d'usage que les quartz étudiés ne contenaient pas d'actinides. Le coefficient d'efficacité TL des particules alpha, $\mathrm{k}$, qui est une propriété intrinsèque de la luminescence des matériaux étudiés doit être déterminé et, par ailleurs être pondéré dans notre cas par un paramètre dépendant de la dimension des grains car nous utilisons des quartz de classe granulométrique 80-315 $\mu \mathrm{m}$ sans dissolution des parties externes irradiées par les alpha.
Cela revient à écrire la formule suivante pour la dose d'irradiation annuelle I :

$$
\mathrm{I}=\mathrm{k}^{*} \mathrm{I}_{\alpha}+\mathrm{I}_{\beta}+\mathrm{I}_{\gamma}+\mathrm{I}_{\text {cosmiques }} \quad \text { [Eq. 3] }
$$

où $\mathrm{k}^{*}$ est la valeur effective du coefficient d'efficacité alpha avec $\mathrm{k}^{*}=0,2 \mathrm{k}$ pour des grains de $80 \mu \mathrm{m}$ et $\mathrm{k}^{*}=0,05 \mathrm{k}$ pour des grains de $315 \mu \mathrm{m}$ (Brennan et al., 1991).

La valeur de $\mathrm{k}$, que nous avons supposée constante pour les 3 échantillons, a été mesurée à partir des quartz de l'échantillon BDX 8449 : après broyage, recuit 1 heure à $350^{\circ} \mathrm{C}$, les grains de granulométrie comprise entre 3 et $12 \mu \mathrm{m}$ ont été sélectionnés par sédimentation différentielle, puis déposés homogènement dans des coupelles par décantation. La loi d'acquisition de la TL avec l'irradiation alpha (à l'aide d'une source de ${ }^{241} \mathrm{Am}$ débitant $0,21 \pm 0,01 \mathrm{~Gy} / \mathrm{s}$ ) a été déterminée expérimentalement avec une partie des coupelles. D'autres coupelles ont été irradiées par une dose bêta proche de la dose naturelle, 40 Gy. Lintensité de TL correspondante a été comparée à celle provoquée par l'irradiation alpha. Le facteur $k$ obtenu vaut $: k=0,0382 \pm 0,0020$ valeur relativement faible de l'efficacité, mais néanmoins compatible avec le fait que l'échantillon ayant été relativement peu chauffé dans le passé, certains pièges profonds restent partiellement saturés par l'irradiation géologique rendant la capture des électrons déplacés par les alpha moins probable.

\section{Détermination de la dose anNuelle D'IRRADIATION}

\subsection{Mesure des teneurs en radioéléments et état d'équilibre des séries de l'uranium}

Les teneurs en radioéléments $\mathrm{K}$, $\mathrm{U}$, Th (Tableau 3) ont été déterminées par spectrométrie gamma à bas bruit de fond (Guibert et al., 1997). U $\left({ }^{238} \mathrm{U}\right)$ correspond à la teneur en uranium calculée à partir d'émetteurs de tête de chaîne en équilibre avec ${ }^{238} \mathrm{U}$ comme ${ }^{234} \mathrm{Th}$ ou en rapport isotopique constant, comme ${ }^{235} \mathrm{U}$. U $\left({ }^{226} \mathrm{Ra}\right)$ correspond à la teneur en uranium calculée à partir des raies de ${ }^{214} \mathrm{~Pb}$ et de ${ }^{214} \mathrm{Bi}$ dans les conditions de l'équilibre entre ${ }^{226} \mathrm{Ra}$ (radium-226) et ${ }^{222} \mathrm{Rn}$ (radon-222).

Nous remarquons (Tableaux 3 et 4 ) un déséquilibre dans les chaînes de l'uranium, lié à la mobilité d'éléments solubles de longue période comme le radium ou l'uranium (le thorium est considéré généralement comme immobile en raison de l'extrêmement faible solubilité de ses ions). Ce déséquilibre, caractérisé par une activité de ${ }^{226} \mathrm{Ra}$ inférieure à celle de ${ }^{238} \mathrm{U}$, est d'autant plus marqué sur les granites 


\begin{tabular}{|c|c|c|c|c|c|}
\hline Échantillon & Nature matériau & $\mathbf{K}(\mathbf{\%})$ & $\mathbf{U}\left({ }^{\mathbf{2 3 8}} \mathbf{U}\right) \mathbf{( p p m )}$ & $\mathbf{U}\left({ }^{\mathbf{2 2}} \mathbf{R a}\right)(\mathbf{p p m})$ & $\mathbf{T h}(\mathbf{p p m})$ \\
\hline BDX 8449 & Granite chauffé & $3,47 \pm 0,05$ & $6,38 \pm 0,2$ & $4,21 \pm 0,05$ & $9,21 \pm 0,13$ \\
\hline BDX 8451 & Granite chauffé & $3,13 \pm 0,03$ & $8,75 \pm 0,32$ & $5,56 \pm 0,06$ & $9,56 \pm 0,12$ \\
\hline BDX 8452 & Granite chauffé & $4,08 \pm 0,05$ & $8,02 \pm 0,23$ & $5,33 \pm 0,05$ & $11,84 \pm 0,15$ \\
\hline BDX 8454 & Terre de remplissage & $4,20 \pm 0,06$ & $7,45 \pm 0,24$ & $5,57 \pm 0,06$ & $8,22 \pm 0,13$ \\
\hline BDX 8456 & Granite non chauffé & $4,14 \pm 0,05$ & $7,06 \pm 0,23$ & $5,91 \pm 0,06$ & $10,47 \pm 0,14$ \\
\hline
\end{tabular}

Tableau 3 : Teneurs en radioéléments du granite de Javols et de la terre environnante.

Table 3: Radioelement contents of granite and soil samples from Javols.

\begin{tabular}{|c|c|c|c|c|}
\hline Échantillon & matériau & $\left.\mathbf{U ~}{ }^{(238} \mathbf{U}\right) / \mathbf{T h}$ & $\mathbf{U}\left({ }^{(226} \mathbf{R a}\right) / \mathbf{T h}$ & $\left.\mathbf{U}{ }^{(238} \mathbf{U}\right) / \mathbf{U ~}\left({ }^{226} \mathbf{R a}\right)$ \\
\hline BDX 8449 & granite chauffé & $0,693 \pm 0,024$ & $0,457 \pm 0,008$ & $1,516 \pm 0,050$ \\
\hline BDX 8451 & id. & $0,915 \pm 0,035$ & $0,582 \pm 0,010$ & $1,574 \pm 0,060$ \\
\hline BDX 8452 & id. & $0,677 \pm 0,021$ & $0,450 \pm 0,007$ & $1,504 \pm 0,046$ \\
\hline BDX 8454 & terre remplissage & $0,906 \pm 0,033$ & $0,677 \pm 0,013$ & $1,338 \pm 0,045$ \\
\hline BDX 8456 & granite non chauffé & $0,674 \pm 0,024$ & $0,564 \pm 0,009$ & $1,195 \pm 0,041$ \\
\hline
\end{tabular}

Tableau 4 : Étude du déséquilibre des séries de l'uranium du granite de Javols par l'analyse des rapports de teneurs inter-éléments. Table 4: U-series disequilibrium study of granite and soil samples: ratios of radioelement contents (w/w).

chauffés qu'ils ont été fissurés par le chauffage (Tableau 4) et soumis à des altérations plus intenses que la roche-mère (BDX 8456). Bien qu'un certain degré d'hétérogénéité apparaisse au sein des divers prélèvements analysés, on constate que la teneur en uranium déterminée à partir de l'activité du radium $\mathrm{U}\left({ }^{226} \mathrm{Ra}\right)$ du granite non chauffé, le moins altéré, est la plus forte, indice en faveur d'un lessivage du radium, plutôt que d'un enrichissement en uranium. Par ailleurs, aucun déséquilibre n'a été observé dans la série de ${ }^{232} \mathrm{Th}$, ce qui est lié à la courte période de ${ }^{228} \mathrm{Ra}(5,75 \mathrm{a})$ et de ${ }^{228} \mathrm{Th}$ $(1,91 \mathrm{a})$ (Chu et al., 1999) et à la relative lenteur des processus géochimiques.

\subsection{Une étude de l'homogénéité radiochimique du granite : considérations sur la microdosimétrie}

On s'est interrogé sur la représentativité des mesures de spectrométrie gamma qui portent sur la globalité d'un prélèvement ou sur un échantillon homogénéisé (de plusieurs dizaines de grammes), vis-à-vis de la situation réelle locale de l'irradiation des quartz utilisés en TL, de granulométrie comprise entre 80 et $315 \mu \mathrm{m}$, classe granulométrique très minoritaire (la dimension des grains est plutôt pluri-millimétrique, voire centimétrique). L'existence d'un biais dans les mesures a donc été recherchée en raison de l'hétérogénéité intrinsèque du granite, dont on peut distinguer aussi les effets à large échelle par la variabilité des teneurs en K, $\mathrm{U}$ et Th sur les différents échantillons, pourtant issus de la même roche.
Afin de vérifier ou d'infirmer la représentativité des mesures globales de spectrométrie gamma vis-à-vis de l'irradiation locale des quartz de 80-315 $\mu \mathrm{m}$, nous avons étudié la répartition du potassium autour d'un échantillonnage de ces minéraux sur des lames de granite (BDX 7970 et 8455), par microanalyse $\mathrm{X}$ au microscope électronique à balayage (MEB). En dehors de la facilité de détection de K, son importance dans l'irradiation bêta des quartz est très largement prépondérante (62 à $73 \%$ du total des irradiations alpha et bêta selon l'échantillon).

Des quartz de la dimension requise ont été repérés sur les images (Fig. 3) et pour chacun des spectres d'émission X ont été enregistrés sur des zones carrées de $4 \mathrm{~mm}$ de côté centrées sur le minéral repéré, ces dimensions d'analyse correspondant approximativement à la zone d'influence des particules bêta du potassium- 40 .

Sur deux prélèvements de granite, BDX 7970 et BDX 8455 , nous avons répété ceci une dizaine de fois dans le but d'évaluer la distribution du potassium autour des quartz de 80-315 $\mu \mathrm{m}$ de dimensions. L'étude de répartition montre la variabilité des environnements locaux selon les grains de quartz analysés (Fig. 4) : la majorité possède un environnement dont la teneur en $\mathrm{K}$ est comprise entre 2 et $5 \%$, un grain s'écarte de cette distribution moyenne par un environnement constitué presque exclusivement de feldspath potassique. Aussi, une analyse grain par grain montre-t-elle la grande variabilité de l'irradiation bêta par le seul potassium- 40 dans cette roche. La TL faisant intervenir pour chaque aliquot un grand nombre de grains, il est préférable de considérer les environnements moyens selon l'échantillon. 


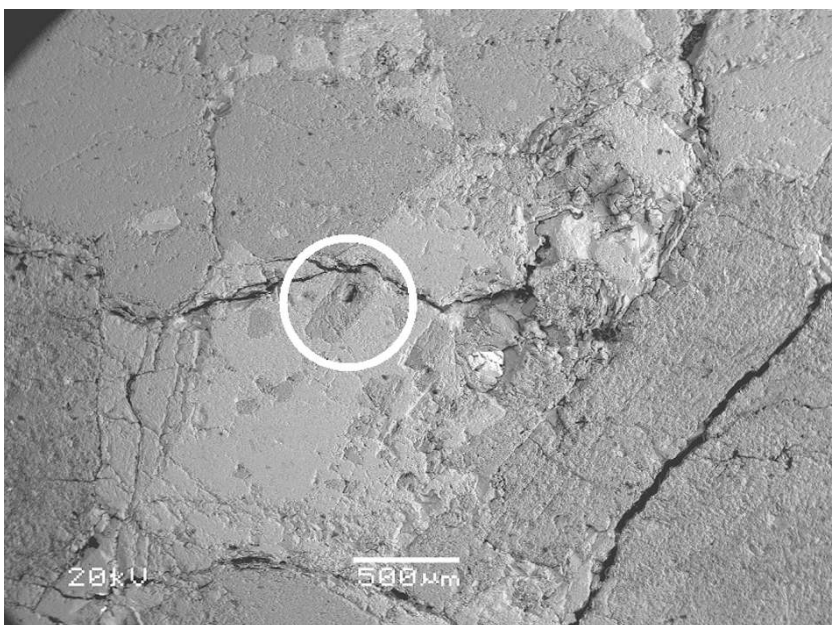

Figure 3 : Microscopie électronique : image en électrons rétrodiffusés d'une lame de granite (BDX 8455) et sélection d'un cristal de quartz de dimensions comprises en 80 et $315 \mu \mathrm{m}$. Le quartz apparaît en gris foncé, feldspaths et micas, de numéro atomique moyen plus élevé que le quartz, apparaissent en gris plus clair.

Figure 3 : SEM backscattered electron image of a section cut into granite sample $B D X 8445$. The target is a quartz grain the size of which ranges between 80 and $315 \mu \mathrm{m}$. Quartz minerals are dark grey; micas, feldspars having a mean atomic number higher than quartz are lighter grey.

Ainsi, les mesures moyennes de spectrométrie $\mathrm{X}$ au MEB (Tableau 5), même si leur écart-type reste élevé en raison de la grande variabilité locale de composition, montrent que les quartz 80-315 $\mu \mathrm{m}$ ont un environnement potassique voisin de ce que l'on peut déduire des mesures globales de spectrométrie gamma à bas bruit de fond.

Bien que présents probablement aussi dans les micas, ainsi que dans des minéraux peu abondants et dispersés (apatite, ilménite...), les actinides ( $\mathrm{U}$ et $\mathrm{Th}$ ) sont surtout présents

\begin{tabular}{|c|c|c|}
\hline Échantillon & matériau & K (\%) \\
\hline BDX 8455 & \multirow{3}{*}{ Granite non chauffé } & $4,29 \pm 0,66$ (MEB) \\
\cline { 1 - 1 } BDX 8456 & & $4,14 \pm 0,05$ (spectro gamma) \\
\hline BDX 8449 & & $3,16 \pm 0,05$ (spectro gamma) \\
\cline { 1 - 1 } BDX 8451 & \multirow{2}{*}{ Granite chauffé } & $3,13 \pm 0,03$ (spectro gamma) \\
\cline { 1 - 1 } BDX 7970 & & $3,20 \pm 0,27$ (MEB) \\
\hline
\end{tabular}

Tableau 5 : Comparaison des teneurs en potassium des échantillons de granite de Javols entre la moyenne des environnements locaux des quartz 80-315 $\mu \mathrm{m}$ et la mesure globale de spectrométrie gamma à bas bruit de fond. NB : l'incertitude représente l'écart type sur la moyenne des mesures locales au MEB alors qu'elle représente l'incertitude classique de mesure en spectrométrie gamma. Table 5: Comparison between global K content measured by gamma spectroscopy and average K content from local analyses by SEM of millimetric surroundings of 80-315 $\mu \mathrm{m}$ grain size quartz. Uncertainty is one standard deviation on $K$ content average value from SEM-EDX measurements, and classical counting statistics standard deviation from gamma spectroscopy.

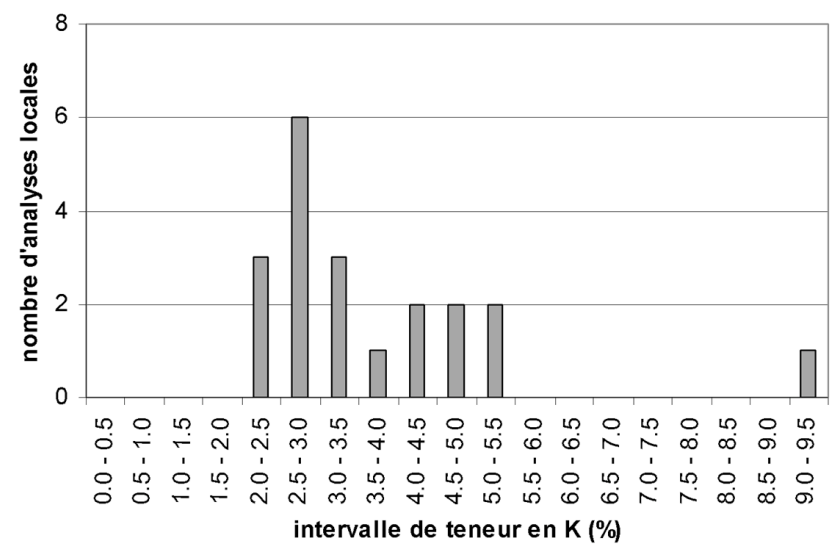

Figure 4 : Histogramme de répartition des teneurs en $\mathrm{K}$ de l'environnement local de grains de quartz $80-315 \mu \mathrm{m}$ dans le granite de Javols.

Figure 4: Distribution of $K$ content of local environment of the 80315 um quartz grains.

dans la fraction fine du granite comme le montre une mesure spécifique par spectrométrie gamma des minéraux de moins de $80 \mu \mathrm{m}$ de l'un des échantillons (BDX 8452) : 31 $\pm 1 \mathrm{ppm}$ $\mathrm{U}\left({ }^{238} \mathrm{U}\right), 16,7 \pm 0,3 \mathrm{ppm} \mathrm{U}\left({ }^{226} \mathrm{Ra}\right)$ et $36,2 \pm 0,6 \mathrm{ppm}$ Th. Il est donc légitime de s'interroger aussi sur la répartition de ces minéraux fins au voisinage des grains de quartz 80-315 $\mu \mathrm{m}$. L'examen qualitatif des images MEB ne montre aucune accumulation particulière de minéraux fins à leur voisinage, plutôt constitué d'un assemblage classique de feldspaths, de quartz et de mica.

Dans l'hypothèse (non testée) où les grains de quartz examinés sur les lames représentent correctement ceux qui ont été prélevés pour l'étude de TL par désagrégation de la roche, et vus les résultats des analyses de répartition qui précèdent, nous avons considéré que les mesures globales de $\mathrm{U}$, de Th et de K étaient également représentatives de leur irradiation locale, alpha et bêta.

\subsection{Détermination de la dose annuelle d'irradiation}

Les composantes alpha et bêta de la dose annuelle (Tableau 7) ont été déterminées à partir des mesures de spectrométrie gamma à bas bruit de fond en appliquant les facteurs de conversion teneurs/dose annuelle tabulés par Adamiec et Aitken (1998), modifiés pour tenir compte du déséquilibre des séries de l'uranium lié au lessivage du radium (Tableau 6). On a de surcroît tenu compte des facteurs d'atténuation de dose bêta et alpha liés à la dimension des grains (Mejdahl, 1979; Brennan et al., 1991). 


\begin{tabular}{|c|c|c|c|c|c|c|c|}
\hline \multirow{2}{*}{ radio-élément } & \multirow{2}{*}{$\begin{array}{c}\text { unité de } \\
\text { concentration }\end{array}$} & \multicolumn{3}{|c|}{$\begin{array}{c}\text { dose annuelle spécifique (mGy/a par } \\
\text { unité de concentration) }\end{array}$} & \multirow{2}{*}{$\begin{array}{c}\text { ensemble } \\
\text { d'isotopes pris en } \\
\text { compte }\end{array}$} & \multirow{2}{*}{$\begin{array}{l}\text { atténuation bêta } \\
80 \mu \mathrm{m}\end{array}$} & \multirow{2}{*}{$\begin{array}{l}\text { atténuation bêta } \\
315 \mu \mathrm{m}\end{array}$} \\
\hline & & alpha & bêta & gamma & & & \\
\hline $\mathrm{U}\left({ }^{238} \mathrm{U}\right)$ & ppm & 0,9563 & 0,0604 & 0,004 & $\begin{array}{l}\text { U238-Th230 } \\
\text { (U235-Pb207) }\end{array}$ & \multirow{2}{*}{0,875} & \multirow[t]{2}{*}{0,74} \\
\hline $\mathrm{U}\left({ }^{226} \mathrm{Ra}\right)$ & ppm & 1,824 & 0,086 & 0,109 & Ra226-Pb206 & & \\
\hline Th & ppm & 0,732 & 0,0273 & 0,0476 & Th232-Pb208 & 0,92 & 0,80 \\
\hline $\mathrm{K}$ & $\%$ & & 0,782 & 0,243 & K40-Ca40,Ar40 & 0,975 & 0,89 \\
\hline
\end{tabular}

Tableau 6 : Composantes de la dose annuelle des échantillons de granite de Javols. La dose annuelle dépendant de la dimension des grains, nous avons reporté les valeurs calculées pour les limites granulométriques choisies. Les facteurs d'atténuation des composantes alpha et bêta de la dose annuelle ont été pris en compte à partir des données de Brennan et al. (1991) et de Mejdahl (1979). La valeur finale retenue comme dose annuelle est la médiane, ce qui équivaut à une équipartition en masse des grains dans l'intervalle granulométrique 80-315 $\mu \mathrm{m}$.

Table 6: Annual dose components for 80-315 $\mu \mathrm{m}$ quartz grains extracted from heated granite samples. As dose rate depends on grain size, limit values at the ends of the grain size range selected were reported. Attenuation factors applied to infinite matrix alpha and beta dose rates were taken into account (Mejdahl, 1979; Brennan et al., 1991). Final dose rate is the medial value (equivalent to a uniform mass distribution of grains respect to their size).

\begin{tabular}{|c|c|c|c|c|c|}
\hline échantillons & $\mathbf{k}^{*} \mathbf{I}_{\alpha}(\mathbf{m G} \mathrm{g} / \mathbf{a})$ & $\mathrm{I}_{\beta}(\mathrm{mG} \mathrm{y} / \mathbf{a})$ & $I_{\text {env }}(m G y / a)$ & $I_{\text {total }}(\mathrm{mGy} / \mathrm{a})$ & $\begin{array}{l}I_{\text {total }}(\mathrm{mGy} / \mathrm{a}) \\
\text { val. médiane }\end{array}$ \\
\hline BDX 8449, $(80 \mu \mathrm{m})$ & $0,163 \pm 0,010$ & $3,53 \pm 0,07$ & \multirow{2}{*}{$2,29 \pm 0,06$} & $5,98 \pm 0,10$ & \multirow{2}{*}{$5,74 \pm 0,17$} \\
\hline BDX 8449, $(315 \mu \mathrm{m})$ & $0,041 \pm 0,003$ & $3,16 \pm 0,06$ & & $5,50 \pm 0,09$ & \\
\hline BDX 8451, $(80 \mu \mathrm{m})$ & $0,205 \pm 0,013$ & $3,53 \pm 0,06$ & \multirow{2}{*}{$2,29 \pm 0,06$} & $6,03 \pm 0,10$ & \multirow{2}{*}{$5,76 \pm 0,18$} \\
\hline BDX 8451, $(315 \mu \mathrm{m})$ & $0,051 \pm 0,003$ & $3,15 \pm 0,06$ & & $5,49 \pm 0,10$ & \\
\hline BDX $8452,(80 \mu \mathrm{m})$ & $0,206 \pm 0,014$ & $4,23 \pm 0,07$ & \multirow{2}{*}{$2,29 \pm 0,06$} & $6,73 \pm 0,11$ & \multirow{2}{*}{$6,43 \pm 0,20$} \\
\hline BDX 8452, $(315 \mu \mathrm{m})$ & $0,052 \pm 0,003$ & $3,79 \pm 0,07$ & & $6,13 \pm 0,10$ & \\
\hline
\end{tabular}

Tableau 7 : Dose spécifique annuelle fournie par U, Th et K exprimée en mGy/a et par unité de concentration; les données pour l'uranium ont été calculées à partir des tables d'Adamiec et Aitken (1998) dans le cas d'un déséquilibre lié à la variation de la concentration en radium. On a indiqué les fragments de chaîne radioactive (dont les isotopes sont en équilibre entre eux) pris en compte ainsi que les facteurs d'atténuation de dose annuelle bêta utilisés selon la dimension des grains (d'après Mejdahl, 1979; Brennan et al., 1991).

Table 7: Annual dose rate factors in $m G y / a$ per concentration unit. $U$ data were calculated from Admiec and Aitken data (1998) assuming that a disequilibrium originated in radium variation. Also are reported the sets of nuclides in equilibrium within each part of $U$-series considered and beta attenuation factors (Mejdahl, 1979; Brennan et al., 1991).

Pour le calcul de la contribution des séries U à la dose annuelle, il a été supposé pour chaque échantillon daté, une variation linéaire dans le temps de la concentration en radium-226 et de ses descendants en équilibre, depuis une teneur d'origine jusqu'à celle observée aujourd'hui, $\mathrm{U}\left({ }^{226} \mathrm{Ra}\right)$, conséquence du surcroît d'altérabilité induite par le chauffage. Pour déterminer cette teneur originelle, nous avons considéré que le substrat granitique non chauffé était déjà altéré et présentait un déséquilibre naturel caractérisé par un rapport $\mathrm{U}\left({ }^{238} \mathrm{U}\right) / \mathrm{U}\left({ }^{226} \mathrm{Ra}\right)$ égal à 1,195 représenté par l'échantillon BDX 8456 (Tableau 4). Nous supposerons que ce rapport était le même au moment du chauffage pour l'ensemble des prélèvements datés. Ainsi, la teneur initiale en ${ }^{226} \mathrm{Ra}$ de chaque échantillon est donnée par $\mathrm{U}\left({ }^{238} \mathrm{U}\right) / 1,195$.
Par contre, l'activité des isotopes de tête de chaîne, de ${ }^{238} \mathrm{U}$ à ${ }^{230} \mathrm{Th}$ et de ${ }^{235} \mathrm{U}$ à ${ }^{207} \mathrm{~Pb}$ respectivement pour les séries de ${ }^{238} \mathrm{U}$ et de ${ }^{235} \mathrm{U}$, a été considérée comme constante dans le temps et égale à celle déduite de $\mathrm{U}\left({ }^{238} \mathrm{U}\right)$.

L'état d'humidité moyen archéologique a été estimé à $1,2 \pm 0,6 \%$ (\% de masse d'eau supposée introduite dans les pores relativement à la masse de l'échantillon sec), ce qui correspond à la demie-saturation pour cette roche.

La dose environnementale, comprenant les composantes gamma et cosmique, a été déterminée par spectrométrie gamma in situ à l'aide d'un scintillateur NaI:Tl (Canberra Inspector, 2,5” x 2,5”). Cette composante a été supposée constante dans le temps. 


\section{Datation}

Les âges-TL des prélèvements de granite sont reportés au tableau 8 avec leurs écarts-types associés. Les fragments appartenant à la même structure, devraient, s'il n'y avait pas d'incertitudes de mesure, fournir le même âge. L'existence d'incertitudes analytiques liées à la fois à la nature des grandeurs physiques mesurées et à la réponse de ces échantillons-là génère une dispersion. Nous avons testé la normalité statistique de cette dispersion, autement dit, nous avons cherché à savoir si l'écart-type affecté aux âges-TL (l'écart-type est calculé à partir des données expérimentales) pouvait rendre compte de la dispersion observée entre 7110 et 5350 ans. Il apparaît que la distribution de ces résultats est statistiquement normale, compte tenu des incertitudes de mesure : le chi-2 vaut 3,60 et appartient à l'intervalle théorique correspondant à $80 \%$ de probabilité $[0,21-4,61]$ (CEA 1978). Nous sommes donc autorisés à effectuer la moyenne pondérée de ces âges individuels; la pondération choisie est inversement proportionnelle à la variance statistique de chaque âge, ce qui conduit à affecter le plus fort poids aux mesures les plus précises, en l'occurrence BDX 8451 puis BDX 8452 et enfin BDX 8449 dans l'ordre d'importance décroissante. La moyenne que nous retenons comme âge-TL final de la chauffe de la structure granitique est de $5825 \pm 280$ ans (incertitude : 1 écart-type), soit un intervalle de confiance compris entre 4380 et 3260 av. J.-C., avec une probabilité de $95 \%$.

Cette datation place la structure de combustion dans un intervalle chronologique qui recouvre le Néolithique final et les débuts du Chalcolithique, pour ce secteur du Massif Central. Il est donc probable que le tesson de céramique trouvé dans la fosse et son fonctionnement comme structure de combustion soient assez proches dans le temps. Cette datation est également compatible avec les indices de la présence des Néolithiques à Javols, repérée par le matériel lithique diffus retrouvé lors de sondages effectués dans la

\begin{tabular}{|c|c|c|}
\hline Échantillon & $\begin{array}{c}\text { âge-TL } \pm \text { écart-type } \\
\text { global }\end{array}$ & $\begin{array}{c}\text { écart-type } \\
\text { statistique }\end{array}$ \\
\hline BDX 8449 & $7110 \pm 670$ ans & 635 ans \\
\hline BDX 8451 & $5770 \pm 320$ ans & 255 ans \\
\hline BDX 8452 & $5350 \pm 490$ ans & 455 ans \\
\hline moyenne pondérée & $\mathbf{5 8 2 5} \pm \mathbf{2 8 0}$ ans & $\mathbf{2 1 0}$ ans \\
\hline
\end{tabular}

Tableau 8: Javols (Lozère), datation TL de trois échantillons extraits d'une fosse granitique chauffée. L'écart-type global est la somme quadratique des écarts-types statistique et systématique. Table 8: Javols (Lozère, France), TL dating of 3 heated granite samples extracted from an ancient pit. The global standard deviation includes both statistical and systematic components quadratically added. vallée. L'hypothèse de rattachement de la fosse à un oppidum qui aurait précédé de peu la fondation de la cité romaine est donc par la même occasion totalement exclue.

\section{Conclusion}

L'utilisation de la TL a permis de proposer un cadre chronologique à une fosse granitique chauffée. Outre l'intérêt archéologique régional que revêt cette étude, c'est l'apport méthodologique sur lequel nous aimerions insister. Le matériau, un granite, peu usité en datation TL en général, et plus particulièrement pour des périodes où ce sont plus souvent des céramiques qui sont étudiées, présente par sa nature même des problématiques spécifiques. Afin d'éviter le fading inhérent aux feldspaths, nous avons apporté un soin particulier à la sélection des cristaux de quartz en utilisant et en adaptant des techniques de séparation minérale et d'attaque chimique sélective. Pour la détermination de la dose archéologique, comme on le fait désormais systématiquement, nous avons déterminé les conditions de recuit en laboratoire des cristaux qui permettent de retrouver les propriétés de luminescence que le matériau devait posséder à l'issue de son chauffage ancien. Cette étape est particulièrement importante dans le cas des quartz, en raison de fortes variations des caractéristiques de TL avec les conditions de recuit. Enfin, pour ce qui concerne l'évaluation de la dose annuelle, nous avons examiné le problème posé par d'éventuelles hétérogénéités locales de l'irradiation des quartz: l'étude réalisée par microscopie électronique a montré l'importante variabilité de l'irradiation bêta par le potassium, cependant, en moyenne, l'environnement potassique local des cristaux de quartz de mêmes dimensions que ceux sélectionnés pour la TL s'accorde avec ce que l'on déduit des mesures globales de spectrométrie gamma, ce qui permet de garantir la représentativité des mesures de radioactivité et par conséquent de valider la datation.

\section{Remerciements}

Ce travail a été soutenu financièrement (fonctionnement et équipements) par la Communauté de Communes de la Terre de Peyre, le CNRS, l'Université de Bordeaux 3, le Conseil Régional d'Aquitaine. Remerciements particuliers à Benoît Ode (conseil général de Lozère jusqu’en 2003) pour le travail sur le terrain. 


\section{Bibliographie}

Adamiec, G. et Aitken, M. J., 1998. Dose rate conversion factors : update, Ancient TL, 16, p. 37-49.

AitKen, M. J., 1985. Thermoluminescence dating. Academic press (England), 359 p.

Brennan, B. J., Lyons, R. G. et Philips, S. W., 1991. Attenuation of alpha particle track dose for spherical grains, Nuclear Tracks and Radiation Measurements, 18, p. 249-253.

CEA (collectif anonyme), 1978. Statistique appliquée à l'exploitation des mesures, t. 1, Paris, Masson, 148 p.

Chu, S. Y., Nordberg, H., Firestone, R. B. et Ekström, L. P., 1999. logiciel Isotope Explorer, US Dpt of Energy, Nuclear Physics Division, données consultées en septembre 2005.

Duttine, M., Guibert, P., Perraut, A., Lahaye, C., Bechtel, F. et Villeneuve, G., 2005. Effects of thermal treatments on TL and EPR of flints and their importance in TL-dating : application to french Mousterian sites of Les Forêts (Dordogne) and Jiboui (Drôme), Radiation Measurements, 39/4, p. 375-385.

FERDIÈre, A., 2003. Rapport final de synthèse, programme pluriannuel d'évaluation du site archéologique de Javols (1996-2003), Tours, vol. 1, 192 p.

Ferdière, A., Ode, B., 2001. Programme collectif de recherche "Evaluation du site de Javols (1996-2003), Rapport d'activité 2001, UMR Arch. et Territ., Tours, 56 p.

Ferdière, A., Fages, G., Guibert, P., Grici, S. et Bechtel, F., 2006. Une fosse du Néolithique final ou du Chalcolithique à Javols (Lozère) et sa datation par thermoluminescence, Préhistoire du Sud Ouest,13/2006-1, p. 99-110.

Ferdière, A. et OdE, B., 2003. Javols/Anderitum, dossier sur les capitales éphémères, L'archéologue, 66, p. 14-16.

FLeming, S. J., 1970. Thermoluminescence dating : refinement of the quartz inclusions method, Archaeometry, 12, 133-146.

Fleming, S. J., 1978. The quartz inclusion method, PACT, 2, p. $125-130$.

Guibert, P., Vartanian, E., Bechtel, F. et Schvoerer, M., 1996. Non linear approach of TL response to dose: polynomial approximation, Ancient TL, 14, n 2, p. 7-14.

Guibert, P., Bechtel, F. et Schvoerer, M., 1997. Déséquilibre des séries de l'uranium, implications sur la dose annuelle en datation par thermoluminescence : une étude à la Grotte XVI,
Cénac et Saint-Julien, Dordogne (France), Quaternaire, 8, p. 377-389.

Hutt, G. et Smirnov, A., 1983. Thermoluminescence dating of sediments by means of the quartz and feldspar inclusion methods, PACT, 9, p. 463-471.

MejDahl, V., 1979. Thermoluminescence dating : beta-dose attenuation in quartz grains, Archaeometry, 21, 1, p. 61-72.

MejDahl, V., 1982. An automated procedure for thermoluminescence dating of pottery and burnt stones, PACT, 7, p. 83-96.

Mejdahl, V. et Winther-Nielsen, M., 1982. TL dating based on feldspar inclusions, PACT, 6, p. 426-437.

Roque, C., Guibert, P., Vartanian, E., Vieillevigne, E. et Bechtel, F., 2004a. Changes in luminescence properties induced by thermal treatments; a case study at Sipan and Trujillo Moche sites (Peru), Radiation Measurements, 38, p. 119-126.

Roque, C., Guibert, P., Duttine, M., Vartanian, E., Chapoulie, R. et BeChtel, F., 2004b. Dependence of luminescence characteristics of irradiated quartz with thermal treatment and consequences for TL-dating: Application to Dating of Solutrean heated quartz pebbles from Laugerie Haute (Dordogne, France), Geochronometria, 23, p. 1-8.

Sanderson, D. C. W., 1988. Fading of TL in feldspars : characteristics and corrections, Nuclear Tracks and Radiation Measurements, 14, p. 155-161.

Sanzelle, S., Miallier, D., Pilleyre, Th., Faïn, J. et Montret, M., 1996. A new slide technique for regressing TL/ESR dose response curves, Radiation Measurements, 26, p. 631-638.

Vieillevigne E., Guibert P., Bechtel F. et Leriche P., 2004. Thermoluminescence et chronologie de l'architecture médiévale : datation de briques de la citadelle de Termez en Ouzbékistan, Revue d'Archéométrie, vol. 28, p. 43-55.

Wintle, A. G., 1973. Anomalous fading of TL in mineral samples, Nature, 245, 143-144.

ZimmermanN, D. W., 1971. Thermoluminescence dating using fine grains from pottery, Archaeometry, 13, p. 29-52.

ZINK, A., 1996. Thermoluminescence des feldspaths : Emission par effet tunnel et par thermoluminescence dans l'infra-rouge, incidences sur la datation des feldspaths, thèse de doctorat des Universités Paris VII Denis Diderot et Bordeaux III Michel de Montaigne, $275 \mathrm{p}$. 